\title{
Microanalyse nucléaire d'aciers nitrurés
}

\author{
Pierre Vigier $\left({ }^{1}\right)$, Constantin Iacob $\left({ }^{1}\right)$ et Daniel David $\left({ }^{2}\right)$ \\ $\left({ }^{1}\right)$ Groupe de Métallurgie Physique $\left({ }^{*}\right)$, Université de Rouen, \\ 76821 Mont-Saint-Aignan Cedex, France \\ $\left({ }^{2}\right)$ Université de Technologie de Compiègne, UTC/LG2MS, Centre de recherche de Royallieu, \\ BP. 649, 60206 Compiègne, France
}

(Reçu le 15 juillet 1997; Accepté le 19 décembre 1997)

PACS.68.35.Dv - Composition, defects and impurities
PACS.81.65.Lp - Surface hardering: nitridation, carburization, carbonitridation
PACS.81.70.Yp - Other methods of materials testing and analysis

Abstract. - Profiles of the nitrogen content into the bulk, were obtained from nuclear microanalysis. These profiles were the result of a nitriding thermochemical treatment of steel samples; the treatment consists of gazeous nitriding classical or using a fluidized bed process. Experimental and modelized results give an explanation of the surface properties.

Résumé. - Les profils de concentration d'azote en profondeur, ont été réalisés en utilisant la microanalyse nucléaire. Ils concernaient des aciers ayant subi un traitement thermochimique de nitruration par voie gazeuse classique et en lit fluidisé. Les résultats obtenus, comparés à une modélisation, permettent d'expliquer la modification des propriétés de surface.

\section{Introduction}

La détermination du profil de concentration en profondeur d'un matériau ayant subi un traitement thermochimique de surface est essentielle pour caractériser l'efficacité du traitement. La corrélation de ce profil avec les microstructures observées est la clef de la connaissance des mécanismes mis en jeu. La concentration superficielle joue un rôle particulier puisqu'elle fixe les conditions aux limites à imposer aux solutions des équations qui régissent le transfert des atomes, de l'atmosphère de traitement vers le cœur de la pièce à traiter. Ces atomes sont très souvent à faible numéro atomique $(\mathrm{N}, \mathrm{C}, \mathrm{B}, \mathrm{O} \ldots)$ et par conséquent les analyses quantitatives mettant en œuvre les rayons X sont peu adaptées. La microanalyse nucléaire offre une solution alternative pertinente pour donner la concentration d'atomes (azote dans notre cas), sur une profondeur de quelques microns sous la surface, avec une précision de l'ordre du pourcent.

Des aciers faiblement alliés XC $38(0,38 \% \mathrm{C}, 0,27 \% \mathrm{Si}, 0,76 \% \mathrm{Mn}, 0,17 \% \mathrm{Cr}, 0,1 \% \mathrm{Ni}$, $0,03 \% \mathrm{~S}, 0,03 \% \mathrm{Al}$ ) à l'état recuit après laminage, ont été nitrurés en présence d'un mélange gazeux (azote, ammoniac) statique (NGC = nitruration en gaz classique) ou en lit fluidisé (NLF). Le plan d'expérience réalisé est indiqué dans le tableau I.

$\left({ }^{*}\right)$ UMR 6634 CNRS 
Table I. - Plan d'expérience indiquant les méthodes et les paramètres de nitruration des échantillons étudiés.

[Experimental parameters values and processes of the studied nitrided samples.]

\begin{tabular}{|c|c|c|c|c|c|c|c|c|}
\hline $\begin{array}{c}\text { Temps et température } \\
\text { de nitruration }\end{array}$ & $1 \mathrm{~h}$ & $2 \mathrm{~h}$ & $4 \mathrm{~h}$ & $6 \mathrm{~h}$ & $1 \mathrm{~h}$ & $2 \mathrm{~h}$ & $4 \mathrm{~h}$ & $6 \mathrm{~h}$ \\
\hline $480^{\circ} \mathrm{C}$ & $\mathrm{A} 1$ & $\mathrm{~A} 2$ & $\mathrm{~A} 3$ & $\mathrm{~A} 4$ & & $\mathrm{E} 1$ & $\mathrm{E} 2$ & $\mathrm{E} 3$ \\
\hline $520^{\circ} \mathrm{C}$ & $\mathrm{B} 1$ & $\mathrm{~B} 2$ & $\mathrm{~B} 3$ & $\mathrm{~B} 4$ & $\mathrm{~F} 2$ & $\mathrm{~F} 3$ & $\mathrm{~F} 4$ & \\
\hline $560^{\circ} \mathrm{C}$ & $\mathrm{C} 1$ & $\mathrm{C} 2$ & $\mathrm{C} 3$ & $\mathrm{C} 4$ & $\mathrm{G} 1$ & $\mathrm{G} 2$ & $\mathrm{G} 3$ & \\
\hline Mode de nitruration & \multicolumn{4}{|c|}{$\mathrm{NLF}$} & \multicolumn{5}{c|}{ NGC } \\
\hline
\end{tabular}

Après avoir rappelé brièvement les bases de la nitruration des aciers et plus particulièrement de la nitruration effectuée en lit fluidisé, les résultats d'analyses nucléaires sont présentés et comparés à des simulations de concentration en profondeur. Rapprochés des conditions expérimentales, les simulations offrent la possibilité de dégager les mécanismes spécifiques de nitruration en lit fluidisé.

\section{Nitruration des aciers}

\subsection{Généralités sur la nitruration $[1,2]$}

On regroupe, sous le terme général de nitruration, l'ensemble des traitements à base d'azote qui, à des températures comprises entre 400 et $700^{\circ} \mathrm{C}$, permettent d'obtenir des microstructures superficielles sensiblement voisines.

Les transformations de phases associées au développement des couches de combinaison et de diffusion pendant la nitruration permettent d'obtenir un important gradient de dureté déterminant la résistance à l'usure. La présence de l'azote depuis la surface jusqu'à une certaine profondeur confère aux matériaux :

- un durcissement superficiel ;

- une forte augmentation de la limite de fatigue ;

- une amélioration sensible de la résistance à la corrosion.

\subsection{Procédés de nitruration}

La solubilité de l'azote dans la structure ferritique ou austénitique d'un acier dans certaines conditions de milieu, de température et de temps, conditionne l'utilisation de différents procédés de nitruration : nitruration gazeuse dite "classique" par dissociation catalytique de l'ammoniac et son procédé dérivé de nitruration à basse pression [3,4], nitruration ionique ou en plasma [5], nitruration en bains de sels et nitruration en lit fluidisé [6-8].

Nitruration gazeuse classique : l'atmosphère de traitement (ammoniac) est établie dans un four étanche, dont la température détermine le taux de dissociation. Pour accélérer la réaction on peut utiliser comme gaz activateur le protoxyde d'azote $\mathrm{N}_{2} \mathrm{O}$; l'ammoniac peut être aussi dilué avec de l'azote (50\% environ). 

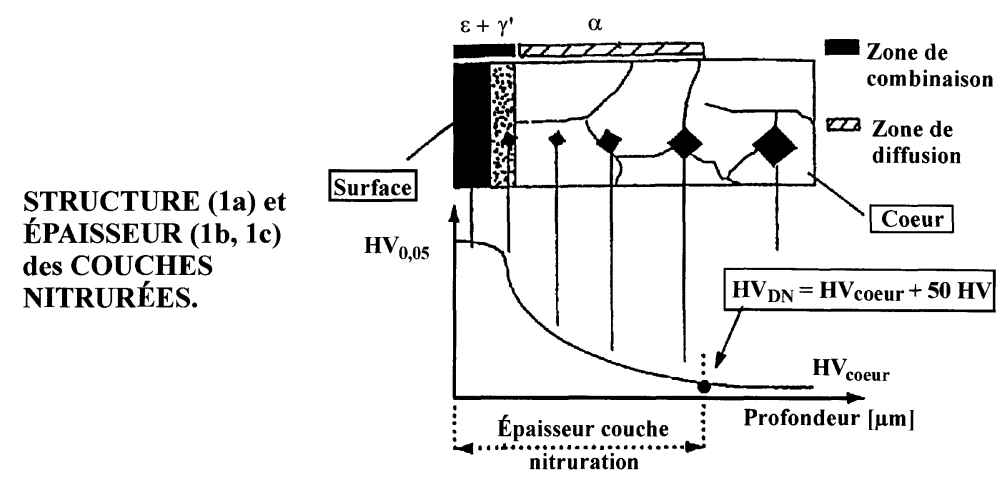

Épaisseur couches (acier XC38)

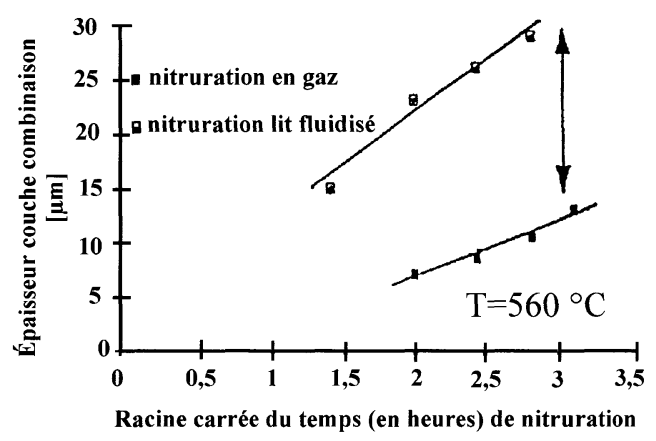

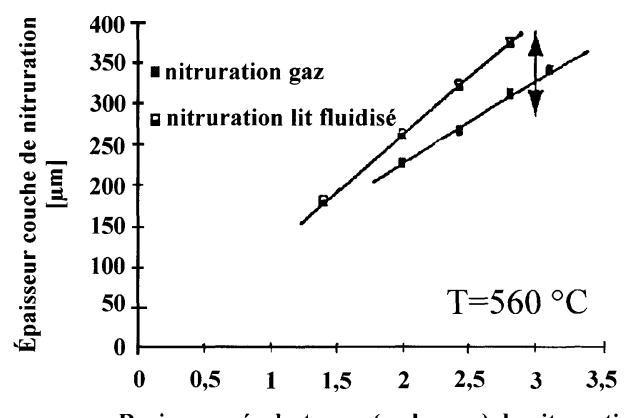

Racine carrée du temps (en heures) de nitruration

Fig. 1. - Croissance des couches de combinaison et de diffusion en fonction du temps de nitruration. [Increasing of the conbination and diffusion layer's thichness versus nitriding time.]

Nitruration en lit fluidisé : le gaz nitrurant est utilisé comme fluidisant d'un sable inerte ; cette technique permet d'augmenter de façon notable l'efficacité de la voie gazeuse classique. Un flux gazeux $\left(\mathrm{NH}_{3}+\mathrm{N}_{2}\right)$, après traversée d'un diffuseur, fluidise un sable (grains d'alumine de diamètre moyen de $150 \mu \mathrm{m}$ ) dans lequel est plongé le matériau à nitrurer. L'ensemble est placé dans un four régulé permettant de travailler aux températures de nitruration, de l'ordre de $550^{\circ} \mathrm{C}$. Le coefficient d'échange thermique entre le lit fluidisé et une sonde immergée dans celui-ci dépend de la température du lit, de la taille des grains de sable, du débit gazeux, des dimensions de la sonde métallique.

\section{Comparaison des nitrurations gazeuses classique et en lit fluidisé}

$\mathrm{Au}$ cours de la nitruration, de nouvelles phases : solutions solides, nitrures et carbonitrures se développent à partir de la surface des aciers traités. On distingue une zone superficielle de combinaison, constituée principalement de nitrures $\left(\varepsilon-\mathrm{Fe}_{2-3} \mathrm{~N}, \gamma^{\prime}-\mathrm{Fe}_{4} \mathrm{~N} \ldots\right)$ et carbonitrures de fer, suivie d'une zone de diffusion où l'azote est en solution interstitielle dans la matrice de fer, avec une concentration décroissant régulièrement jusqu'à retrouver la composition de cœur. Les mesures de microdureté effectuées sur des coupes transversales des l'échantillons nitrurés permettent de déterminer l'épaisseur des couches de combinaison et de nitruration (Fig. 1a).

La variation de l'épaisseur de ces couches, en fonction du temps de traitement pour la nitruration gazeuse classique et en lit fluidisé est montrée sur les figures $1 \mathrm{~b}$ et 1c. On observe 

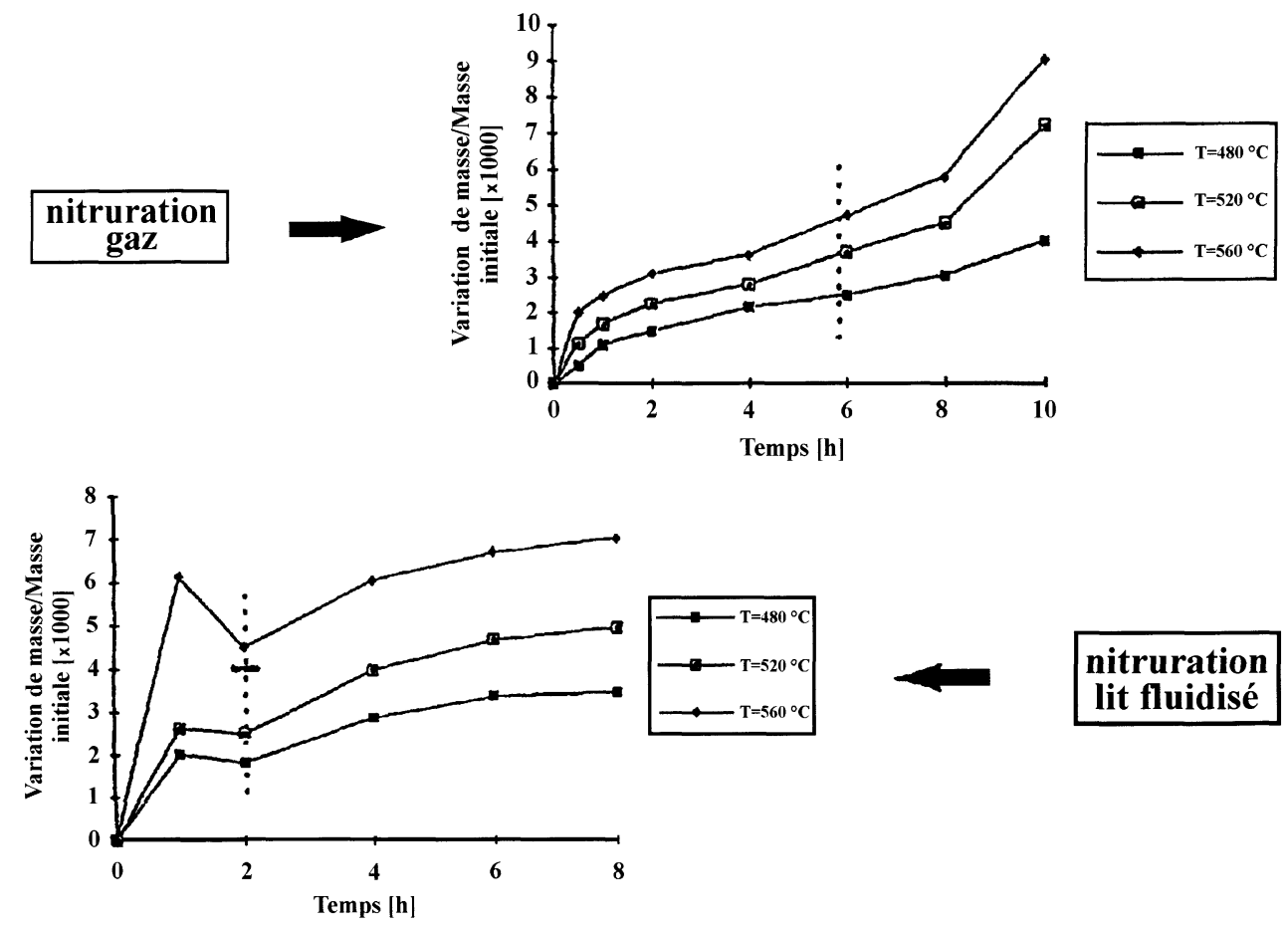

nitruration
lit fluidisé

Fig. 2. - Variation relative de masse après traitement de nitruration en fonction de la durée et de la température de traitement.

[Mass variation due to the nitriding tratment, versus time and temperature of it.]

un écart important de l'épaisseur des couches de combinaison suivant le mode de nitruration ; la cinétique de croissance de cette couche est nettement supérieure en lit fluidisé.

On compare également les deux processus en mesurant la variation de masse des échantillons dans chaque cas (Fig. 2).

On constate systématiquement un enrichissement en azote plus rapide et plus important dans les premiers stades du traitement en lit fluidisé. Pour pouvoir expliquer les raisons de ce comportement, il est nécessaire de connaître de façon quantitative le pourcentage d'azote en fonction de la profondeur et suivre son évolution avec la température et le temps ; nous avons utilisé la microanalyse nucléaire à cet effet. La présence du carbone dans nos échantillons et son comportement au cours de la nitruration feront l'objet d'une étude ultérieure.

\section{Profilométrie d'azote en microanalyse nucléaire}

\subsection{Microanalyse nucléaire [9]}

Un faisceau de particules monocinétiques, accélérées sous des tensions de 0,5 à $3 \mathrm{MeV}$, rencontre la surface d'un matériau et y pénètre à une profondeur de quelques $\mu \mathrm{m}$. Une partie des particules incidentes est rétrodiffusée par les noyaux présents dans la cible (RBS). Une autre partie induit des réactions nucléaires avec les noyaux des éléments légers avec émission de nouvelles particules (Fig. 3) ; le noyau réagissant se transforme en un noyau résiduel qui 


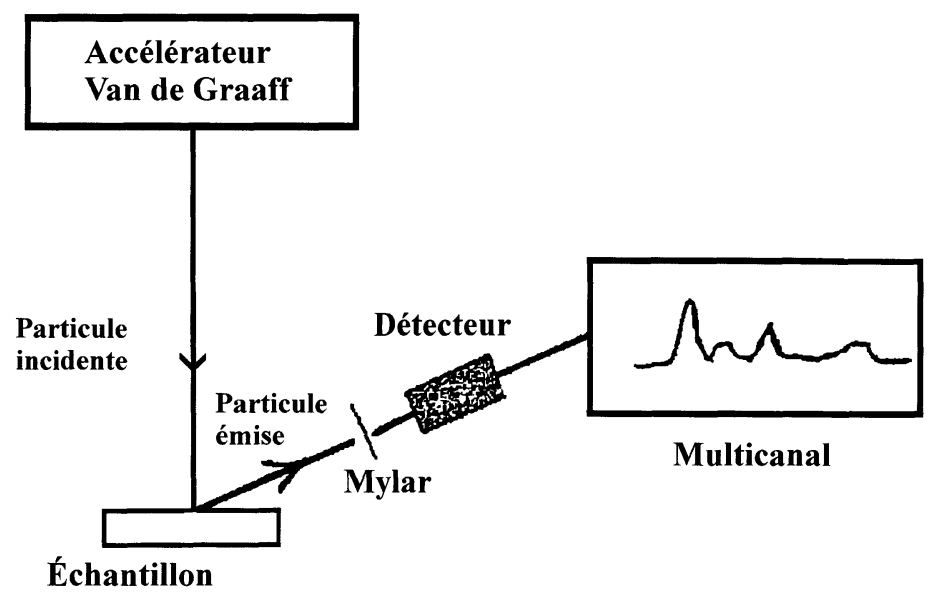

Fig. 3. - Schéma de principe de la microanalyse nucléaire.

[Nuclear microanalysis layout.]

n'intervient pas dans le processus de mesure. Dans notre analyse d'azote, nous avons exploité la réaction : ${ }^{14} \mathrm{~N}(\mathrm{~d}, \alpha){ }^{12} \mathrm{C}$ où des deutons incidents d'énergie $1,2 \mathrm{MeV}$ transforment des noyaux d'azote (14) en carbone (12) avec émission de particules $\alpha$.

L'appareillage utilisé est un accélérateur de type Van de Graaff. La chambre d'analyse renferme des détecteurs solides protégés du flux intense des deutons rétrodiffusés par une feuille de mylar. Les particules à analyser sont captées dans une direction définie par l'axe du détecteur ; elles conduisent à la formation d'un spectre dont les pics correspondent à des états d'excitation caractéristiques des réactions nucléaires.

Le pic utilisé pour déterminer le taux d'azote est situé dans la région haute énergie du spectre. Sa hauteur est proportionnelle à la quantité d'azote, dans l'échantillon, à l'endroit étudié. L'utilisation d'un étalon est nécessaire pour donner les compositions en valeur absolue. La sensibilité de la méthode est de l'ordre de $10^{-3} \mu \mathrm{g} / \mathrm{cm}^{3}$.

La figure 4 correspond à l'analyse d'un échantillon témoin de concentration en azote comprise entre 19,5 at.\% et 19,9 at.\%. Il s'agit d'un nitrure $\mathrm{Fe}_{4} \mathrm{~N}$ obtenu par nitruration d'un fer ARMCO, pendant 6 heures à $550^{\circ} \mathrm{C}$, sous plasma $\mathrm{N}_{2}-\mathrm{H}_{2}$. La hauteur du plateau permet d'étalonner les spectres de nos échantillons.

\subsection{Profil de concentration de la couche de diffusion}

La figure 5 donne le profil global de concentration relative d'azote, de la surface au coeur du matériau ( 0 à $400 \mu \mathrm{m}$ ) dans une tige d'acier XC 38, nitrurée en gaz pendant 8 heures, à des températures variant de 480 à $600^{\circ} \mathrm{C}$. L'allure des courbes suit approximativement la forme des solutions des équations de diffusion de FICK. Le mode d'échantillonnage par découpage de pastilles introduit une incertitude dans le positionnement des surfaces mesurées qui explique aisément les écarts observés entre résultats expérimentaux et lois de FICK ; ceci rend discutables les valeurs de coefficient moyen de diffusion d'azote dans la matrice ferritique que l'on peut calculer $\left(11,6 \times 10^{-8} \mathrm{~cm}^{2} \mathrm{~s}^{-1}\right.$ à $\left.600^{\circ} \mathrm{C}\right)$ à partir de la décroissance de la concentration d'azote avec la profondeur. Pour corriger ce défaut nous avons donc repris cette étude en nous focalisant sur la concentration en azote proche de la surface et son évolution avec les conditions expérimentales : température, temps et type de traitement de nitruration. 


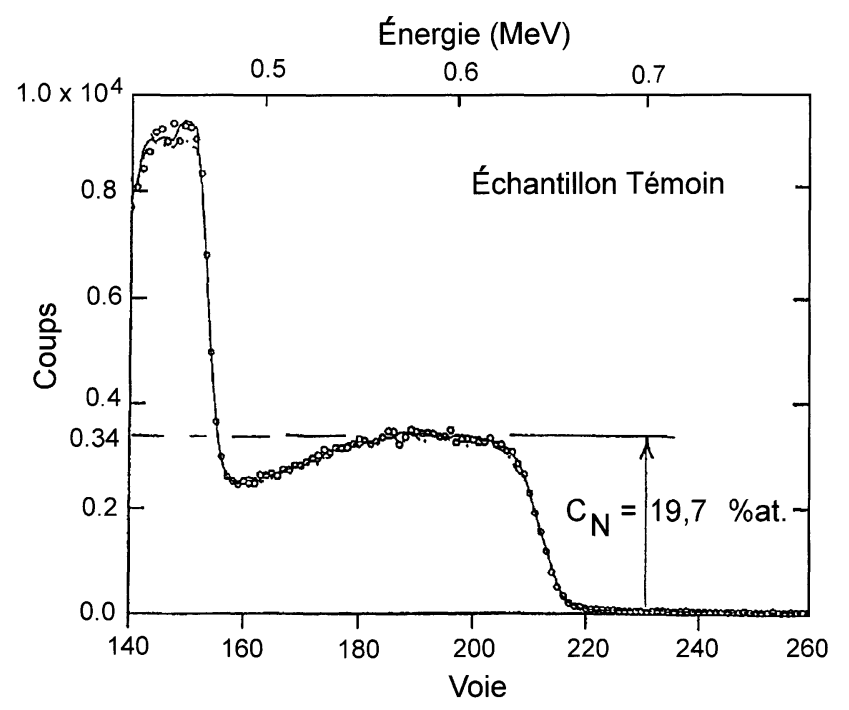

Fig. 4. - Analyse nucléaire d'un échantillon témoin.

[Nuclear analysis of a reference sample.]

\subsection{Analyse nucléaire des concentrations proches de la surface}

Les analyses systématiques effectuées sur les surfaces des échantillons ont donné des résultats que l'on peut grouper schématiquement en trois catégories.

\subsubsection{Spectres en "plateau"}

On les rencontre pour les échantillons A1, A2, E1, F2, G1 c'est-à-dire ceux qui correspondent aux premiers stades de nitruration gazeuse classique ou en lit fluidisé. La figure 6a illustre ce comportement pour l'échantillon A2. Sur une profondeur analysée de l'ordre du micron, on mesure une concentration d'azote de 18,3 at.\% ce qui correspond à un domaine pratiquement monophasé $\gamma^{\prime}$. L'accord entre les résultats expérimentaux $(+)$ et le résultat d'une simulation à concentration uniforme $(-)$ est très correct excepté au voisinage immédiat de la surface (montée abrupte à droite du graphe) ; ceci peut s'expliquer car, à l'échelle de la centaine de nanomètres, la surface présente des irrégularités qui perturbent la réponse en concentration.

\subsubsection{Spectres en "bosse"}

Ce type de réponse est caractéristique des échantillons nitrurés en gaz (classique) excepté E1, F2, G1 (premiers stades de nitruration). Le pic large observé en surface correspond à une croûte superficielle, de concentration décroissant régulièrement à partir de 15 at.\% d'azote (domaine biphasé $\alpha+\gamma^{\prime}$ ) sur 0,5 $\mu$ m (figure 6b, échantillon G2-gaz, $560{ }^{\circ} \mathrm{C}, 2$ heures).

\subsubsection{Spectres en "Rampe"}

On trouve de tels spectres pour les échantillons A, B, C (autres que A1 et A2), nitrurés en lit fluidisé. La figure $6 \mathrm{c}$ correspond à l'échantillon C2 (nitruré en lit fluidisé, pendant 2 heures, à $560^{\circ} \mathrm{C}$ ). Le profil simulé représente une décroissance régulière de la concentration en azote à partir de 21 at.\% (domaine biphasé $\gamma^{\prime}+\varepsilon$ ). 


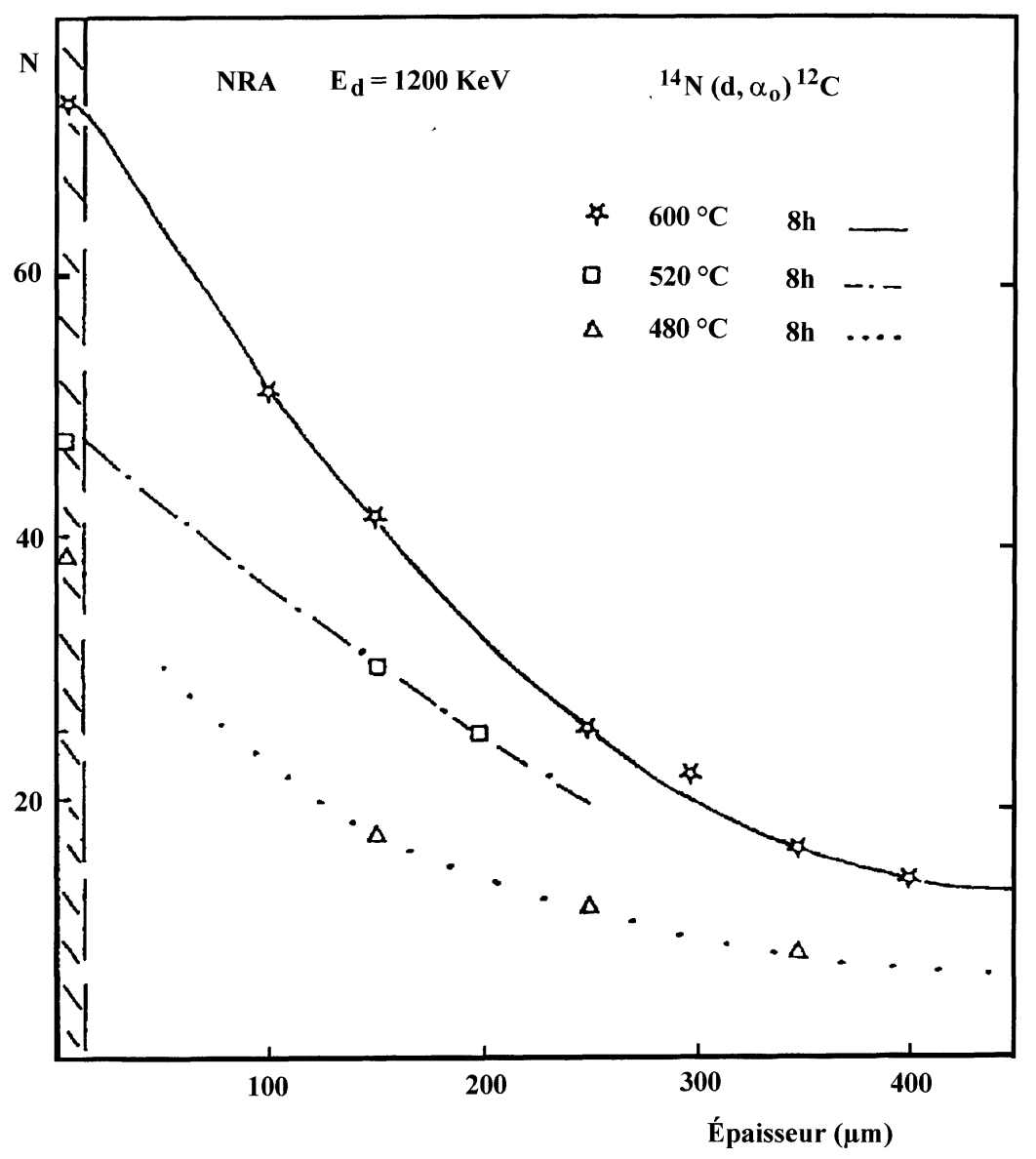

Fig. 5. - Profil de concentration d'azote global en fonction de la profondeur à l'intérieur de la zone de diffusion.

[Nitrogen profile versus depth into the diffusion zone.]

Le domaine exploré s'étend sur une profondeur inférieure à $1 \mu \mathrm{m}$; la couche de combinaison peut atteindre une dizaine de microns et il n'est pas certain que la variation de concentration y suive, de façon lente et continue, l'évolution observée à proximité de la surface ; il faut donc étendre la simulation à une profondeur plus importante [10].

\section{Conclusion}

Le profil de concentration d'azote au voisinage de la surface d'un matériau nitruré est une donnée essentielle à la compréhension des mécanismes de nitruration. Le travail présenté montre la capacité de l'analyse nucléaire à donner cette information. Il apparaît clairement que la composition superficielle dépend fortement du mode et des conditions (température et temps) de nitruration. Les valeurs importantes de concentration observées pour les traitements en lit fluidisé, valeurs qui augmentent avec le temps de traitement alors qu'elles ont plutôt tendance à diminuer dans le cas de la nitruration gazeuse classique, s'expliquent si on admet 

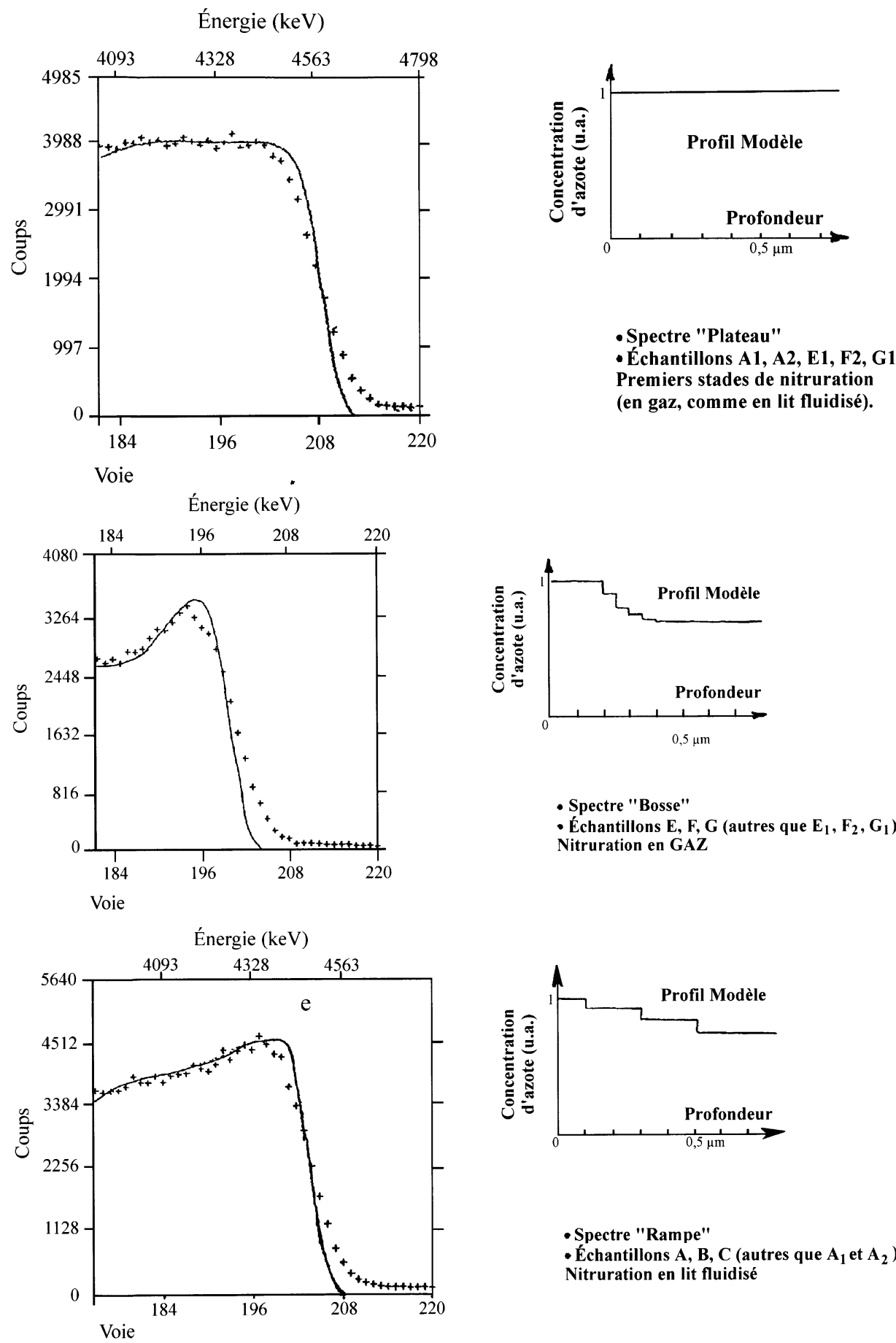

Fig. 6. - Concentration d'azote mesurée et simulée.

[Measured and simulated nitrogen content.]

- Spectre "Bosse"

- Échantillons E, F, G (autres que $E_{1}, F_{2}, G_{1}$ ) Nitruration en GAZ 
que l'impact répété des particules du lit régénère en permanence la surface du matériau traité en détruisant, au fur et à mesure de sa formation, la couche limite séparant l'alliage du gaz environnant. La poursuite du travail de simulation pour couvrir l'ensemble des échantillons du tableau I permettra d'expliciter la formation de la croûte limitant la couche de combinaison ; c'est à travers elle que se font les échanges de matière entre le gaz de traitement et l'intérieur du matériau à traiter ; il est donc essentiel de bien la connaître.

\section{Acknowledgments}

Les auteurs de l'article remercient, pour leur coopération H. Michel, LSGS, École des Mines de Nancy (fourniture du témoin servant à étalonner les spectres en concentration d'azote), les GDR 86 et 1108.

\section{References}

[1] Gantois M., Mechanism and Modelling of Mass Transfert during Gas-Solid Thermochemical Surface Treatment, Second ASM Heat Treatment and Surface Engineering Conference in Europe, Dortmund, 1-3 juin 1993.

[2] Michel H. et Gantois M., Mécanismes de nitruration des alliages ferreux et perspectives d'évolution des procédés de traitement, ATTT La nitruration, Recueil de conférences, Paris, 17 décembre 1993.

[3] CETIM, Choix de traitements thermiques superficiels, Lavoisier, Paris, 1993.

[4] Salvi B., Pittion B. et Pailleaux A., Les nitrurations : choix du procédé en fonction d'application, ATTT, La nitruration, Recueil de conférences, Paris, 17 décembre 1993.

[5] Lebrun J.P., Évolution et développements récents en nitruration ionique, ATTT, La nitruration. Recueil de conférences, Paris, 17 décembre 1993.

[6] Grellet B., Nouveaux développements sur la nitrocarburation en bains de sels activés au soufre, ATTT, La nitruration, Recueil de conférences, Paris, 17 décembre 1993.

[7] Iacob C. et Vigier P., Nitruration en lit fluidisé, application aux aciers XC 38 et 40 CAD 6.12 (I), Trait. Therm. 270 (1994) 33.

[8] Iacob C. et Vigier P., Nitruration en lit fluidisé, application aux aciers XC 38 et 40 CAD 6.12 (II), Trait. Therm. 298 (1997) 51.

[9] David D. et Caplain R., Méthodes usuelles de caractérisation des surfaces, Eyrolles, Paris, 1988.

[10] Sommers A.J. et Mittermejer E.J., Formation de la couche de combinaison de fer lors d'une nitrocarburation gazeuse ou en bains de sels, Trait. Therm. 270 (1994) 27. 